\title{
Propiedades fisicoquímicas durante el crecimiento y desarrollo en dos variedades de frutos de lulo (Solanum quitoense Lam.)
}

\section{Physicochemical properties during growth and development of fruits of two varieties of lulo (Solanum quitoense Lam.)}

PEDRO JOSÉ ALMANZA-MERCHÁN ${ }^{1,3}$ JULIÁN DAVID VELANDIA D. ${ }^{2}$

YEILY PAOLA TOVAR L. ${ }^{2}$

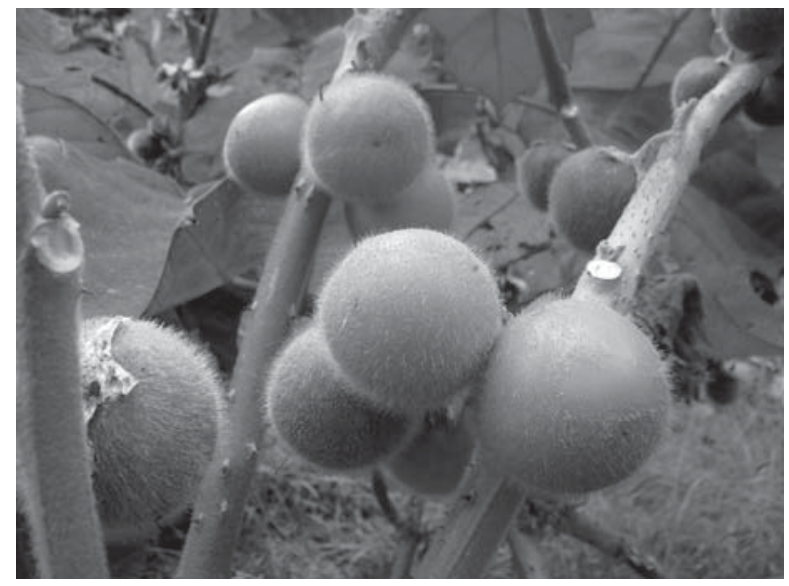

Frutos maduros de lulo.

Foto: P.J. Almanza-Merchán

\section{RESUMEN}

El lulo (Solanum quitoense Lam.) se ha considerado frutal promisorio y especie potencial para mercados globales, constituyéndose en un renglón importante para la economía del departamento de Boyacá (Colombia). La presente investigación buscó realizar una caracterización fisicoquímica de los frutos de dos variedades de lulo (Chonto grueso y Criollo) durante su crecimiento y desarrollo, procedentes del municipio de Pachavita, Boyacá. Para ello se tomaron frutos cada 10 días, a partir de los 30 días después de antesis (dda). Se evaluó la masa fresca, acidez total titulable (ATT), sólidos solubles totales (SST), firmeza y tasa respiratoria (TR). Se encontró que los frutos de las dos variedades presentaron un crecimiento tipo sigmoide simple que se ajustó a un modelo de crecimiento logístico, apreciándose tres fases de crecimiento que se describieron como: Fase I de división celular; Fase II de elongación celular; y Fase III de maduración. Las propiedades químicas se ajustaron a modelos polinómicos, donde la ATT y los SST aumentaron progresivamente, mientras que la TR se redujo siguiendo las características propias de los frutos de lulo, logrando la calidad organoléptica requerida para el consumo.

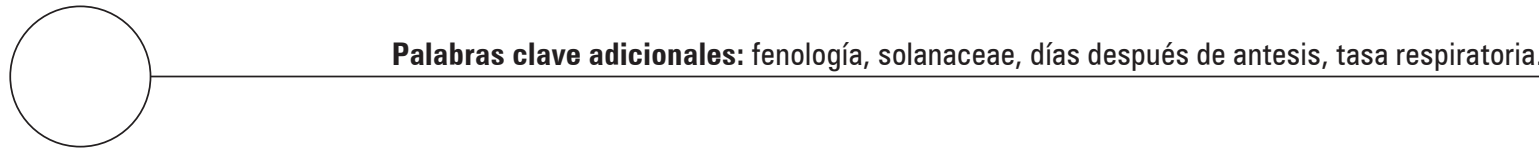

\footnotetext{
1 Facultad de Ciencias Agropecuarias, Grupo de Investigaciones Agrícolas, Universidad Pedagógica y Tecnológica de Colombia (UPTC), Tunja (Colombia).

2 Facultad de Ciencias Agropecuarias, Universidad Pedagógica y Tecnológica de Colombia (UPTC), Tunja (Colombia).

3 Autor para correspondencia.ppcalma@gmail.com
} 


\section{ABSTRACT}

The lulo (Solanum quitoense Lam.), considered a promissory fruit and potential species to global markets, has become an important sector to the economy of Boyacá Department (Colombia). The current investigation seeks to characterize fruits from two varieties of lulo (Chonto grueso and Criollo) during its growth and development, grown in the municipality of Pachavita, Boyaca. For that, each 10 days, fruits of the two varieties were collected starting from 30 days after the anthesis (daa). The physicochemical features of fresh biomass, total titratable acidity (TTA), total soluble solids (TSS), firmness and respiratory rate (RR) were evaluated. It was found that fruits of the two varieties had a sigmoid type growth curve that was adjusted to a logistic growth model, observing three growth phases described as: Phase I, cellular proliferation; Phase II, cellular elongation; and Phase III, ripening. In the same way, the chemical properties were adjusted to polynomial models, where the TTA and TSS increased progressively, whereas the RR was decreasing according to the own characteristics of lulo fruits and the organoleptic quality required for its consumption.

Additional key words: phenology, Solanaceae, days after anthesis, respiration rate.

Fecha de recepción: 13-05-2016 Aprobado para publicación: 24-11-2016

INTRODUCCIÓN

El cultivo de lulo (Solanum quitoense Lam.) se ha convertido en un renglón importante para los agricultores del municipio de Pachavita, por ser un factor esencial en la economía familiar campesina, al constituirse como fuente de ingresos y por ser una alternativa para reemplazar los cultivos ilícitos (Múnera, 2002), y por su potencial agroindustrial (Morales et al., 2002). Del total del área sembrada en Colombia, el $74 \%$ se establece bajo las características de economía campesina, representada una producción de 65.000 t año $^{-1}$ (Andrade et al., 2013), el 26\% restante se cultiva bajo economía empresarial. Su producción se ha incrementado paulatinamente, reportándose 8.418 ha plantadas en Colombia (Agronet, 2015). Además, Arias y Rendón (2014) reportan una tendencia al alza en el comportamiento del precio en Colombia, impulsada por el aumento de la demanda interna; sin embargo, la corta vida del fruto en poscosecha, está determinada por un rápido deterioro de las funciones fisicoquímicos y fisiológicas que acompañan al proceso de maduración (Andrade et al., 2013; Ramírez et al., 2013; Torres et al., 2013), afectando la calidad del fruto.

De otra parte, Cañizares et al. (2003) mencionan que los estudios de crecimiento y desarrollo de frutos son importantes para evaluar las etapas óptimas de maduración, para determinar el comportamiento del crecimiento a través del tiempo, y así, estimar el tamaño del fruto (Avanza et al., 2008) y el peso en la cosecha (Ardila et al., 2011; Coombe, 1976), con lo que se proponen estrategias de manejo agrícola, para finalmente establecer etapas fenológicas y analizar la formación del fruto y su desarrollo estructural (Mazorra et al., 2006). Al respecto, Grange (1996) afirma que el tamaño final de los frutos, está determinado por tres procesos que corresponden a: la multiplicación celular (responsable de la formación del ovario antes de la antesis); seguido de la división celular (después de la antesis y la polinización) y el tercero corresponde a la extensión celular, lo que implica el desarrollo coordinado de un gran número de tejidos (Barceló et al., 2001). Aparte de los factores genéticos, número de semillas por fruto, Fischer et al. (2012a) mencionan influencias externas y de la planta que regulan el tamaño del fruto como el clima, el número y la posición de frutos en la copa, su posición en la inflorescencia, la edad de la planta y el suministro con agua y nutrientes.

Durante el proceso de maduración que ocurre como parte del crecimiento del fruto, en una secuencia de hechos naturales (Gallo, 1996), predominan las reacciones de hidrólisis mediante la cual se desdoblan los carbohidratos (almidón y pectinas) que fueron sintetizados en el fruto en estados tempranos de desarrollo, en moléculas más pequeñas como azúcares solubles y ácidos pécticos; estos procesos se ven reflejados en 
cambios del sabor, consistencia, color y aroma (Parra y Hernández, 2005). A su vez se presentan cambios bioquímicos en la tasa respiratoria, producción de etileno, actividad de la poligalacturonasa, azúcares solubles y los ácidos orgánicos (Balaguera-López y Herrera (2012). Por tanto, el objetivo del presente estudio fue caracterizar el comportamiento de algunas propiedades fisicoquímicas de frutos de lulo de las variedades Chonto grueso y Criollo durante su crecimiento y desarrollo bajo condiciones de Pachavita, Boyacá.

\section{MATERIALES Y MÉTODOS}

Los frutos se recolectaron en la vereda Sacaneca ( $\mathrm{Pa}-$ chavita, Boyacá), a una altitud de $2.148 \mathrm{msnm}$, con temperatura media de $17^{\circ} \mathrm{C}$. Se seleccionaron 15 plantas de las variedades Chonto grueso y Criollo, marcando las flores en antesis. Las plantas tenían una edad de 3 años, con distancia de siembra de $2 \times 1,5 \mathrm{~m}$. El manejo agronómico del cultivo correspondió a los utilizados por el agricultor.

De cada material, se tomaron 4 frutos al azar, cada $10 \mathrm{~d}$ (desde los 30 dda hasta madurez comercial), que fueron llevados en neveras de icopor al laboratorio de Fisiología Vegetal de la Universidad Pedagógica y Tecnológica de Colombia. La tasa respiratoria (TR) y las variables físicas: masa fresca y firmeza fueron medidas desde los 30 dda. Las variables químicas: acidez total titulable (ATT) y sólidos solubles totales (SST) fueron determinadas a partir de los $70 \mathrm{dda}$ (cuando los frutos presentaron jugo para su determinación). La toma de datos se realizó hasta alcanzar la madurez de cosecha comercial. La masa fresca se valoró mediante balanza de precisión de 0,01 g (Ohaus, Parsippany, NJ); la firmeza con un penetrómetro digital PCE-PTR200 (Albacete, España) con aproximación $0,05 \mathrm{~N}$; para determinar la $\mathrm{TR}$, se utilizaron cámaras herméticas de $2 \mathrm{~L}$, que cuentan con sensor infrarrojo de $\mathrm{CO}_{2}$ conectado a un equipo Labquest (Vernier Software \& Technology, Beaverton), en donde se introdujeron los frutos durante $10 \mathrm{~min}$ (con los datos obtenidos de la pendiente, peso fresco de frutos, y volumen de la cámara, se calculó la TR); los sólidos solubles totales (SST) a través de un refractómetro digital Hanna (Clarkson Laboratory and Supply, Chula Vista, CA) (0 a 85\%) con precisión $0,1{ }^{\circ}$ Brix y la acidez total titulable (ATT) de acuerdo con la metodología descrita por la AOAC (AOAC, 1995), mediante la titulación con $\mathrm{NaOH} 0,1 \mathrm{~N}$ hasta $\mathrm{pH}$ 8,2 y se expresó como ácido cítrico.
Se determinó el promedio, el error estándar y se realizó un análisis de varianza, para establecer las diferencias estadísticas entre variedades a una significancia $\leq 0,05$, mediante la utilización del software SPSS $19.0^{\circledR}$. Se graficó el comportamiento de cada una de las variables respecto al tiempo y se determinaron los modelos estadísticos de mayor ajuste. Las tasas de crecimiento y los modelos logísticos fueron establecidos mediante análisis funcional, con la utilización del software SAS v. 9.2.

\section{RESULTADOS Y DISCUSIÓN}

\section{Masa fresca}

La masa fresca siguió una tendencia de curva tipo sigmoide simple para las dos variedades, comportamiento que se ajustó a un modelo de crecimiento logístico (Tab. 1). Este tipo de curva que es característica de los frutos (Salisbury y Ross, 1994), ha sido reportada en tomates 'Sofía', 'Bravona' y 'Granitio' y 'Quindío', bajo condiciones de invernadero (Casierra-Posada et al., 2007).

En el comportamiento de la masa fresca de frutos de lulo Chonto grueso y Criollo (Fig. 1), se aprecian las tres fases de crecimiento que han sido descritas por Salisbury y Ross (1994). Se observó en la fase I (FI) una baja acumulación de masa fresca hasta los 40 dda para la variedad Chonto grueso, acumulando $6,09 \pm 2,12 \mathrm{~g}$, y hasta los 50 dda para la variedad Crio1lo, con 14,31 $\pm 1,46 \mathrm{~g}$, valores atribuidos al predominio de procesos de división celular (Opara, 2000). La fase II (FII) se caracterizó por el llenado del fruto con predominio de la elongación celular y, una reducción de la intensidad respiratoria (Kays, 2004), presentándose un comportamiento ascendente en la acumulación de masa fresca hasta los 110 dda con 79,64 46,50 g para la variedad Chonto, y 63,765 $\pm 2,7$ g para la variedad Criollo. Esto se debe a la ganancia de agua y fotoasimilados, lo cual se explicaría por un aumento en el poder vertedero de los frutos, representado por el aumento en la demanda potencial de los frutos (Marcelis y Heuvelink, 2007). La tercera fase de maduración (FIII), en las dos variedades, tuvo una duración de $20 \mathrm{~d}$, abarcando desde los 110 hasta los $140 \mathrm{dda}$. El peso final de los frutos de lulo en la FIII para el punto de cosecha fue de $80 \pm 4,11 \mathrm{~g}$ para la variedad Chonto grueso, y de 73,3 $\pm 9,85 \mathrm{~g}$ para la variedad Criollo. 


\begin{tabular}{|c|c|c|c|}
\hline Variable & Variedad & Modelo & $R^{2}$ \\
\hline \multirow{2}{*}{ Biomasa fresca } & Chonto grueso & $y=82,1582 / 1+e^{-0,0597 *(d d a-72,6708)}$ & 0,99 \\
\hline & Criollo & $y=89,2962 / 1+e^{-0,0327^{*}(d d a-93,0628)}$ & 0,98 \\
\hline \multirow{2}{*}{ Firmeza } & Chonto grueso & $y=5 E-05 x^{4}-0,0195 x^{3}+2,8002 x^{2}-173,34 x+3988,7$ & 0,94 \\
\hline & Criollo & $y=5 E-05 x^{4}-0,0208 x^{3}+3,0161 x^{2}-188,58 x+4374,4$ & 0,92 \\
\hline \multirow{2}{*}{ Intensidad respiratoria } & Chonto grueso & $y=2 E-05 x^{4}-0,0079 x^{3}+1,0946 x^{2}-64,587 x+1407,4$ & 0,97 \\
\hline & Criollo & $y=7 E-06 x^{4}-0,003 x^{3}+0,464 x^{2}-31,457 x+817,29$ & 0,99 \\
\hline \multirow{2}{*}{ Acidez total titulable } & Chonto grueso & $y=2 E-07 x^{5}-9 E-05 x^{4}+0,0197 x^{3}-2,058 x^{2}+105,77 x-2137,6$ & 0,99 \\
\hline & Criollo & $y=7 E-08 x^{5}-4 E-05 x^{4}+0,0082 x^{3}-0,8421 x^{2}+42,646 x-847,27$ & 0,95 \\
\hline \multirow{2}{*}{ Sólidos solubles totales } & Chonto grueso & $y=3 E-05 x^{3}-0,008 x^{2}+0,7492 x-18,322$ & 0,99 \\
\hline & Criollo & $y=4 E-05 x^{3}-0,0117 x^{2}+1,136 x-32,015$ & 0,95 \\
\hline
\end{tabular}

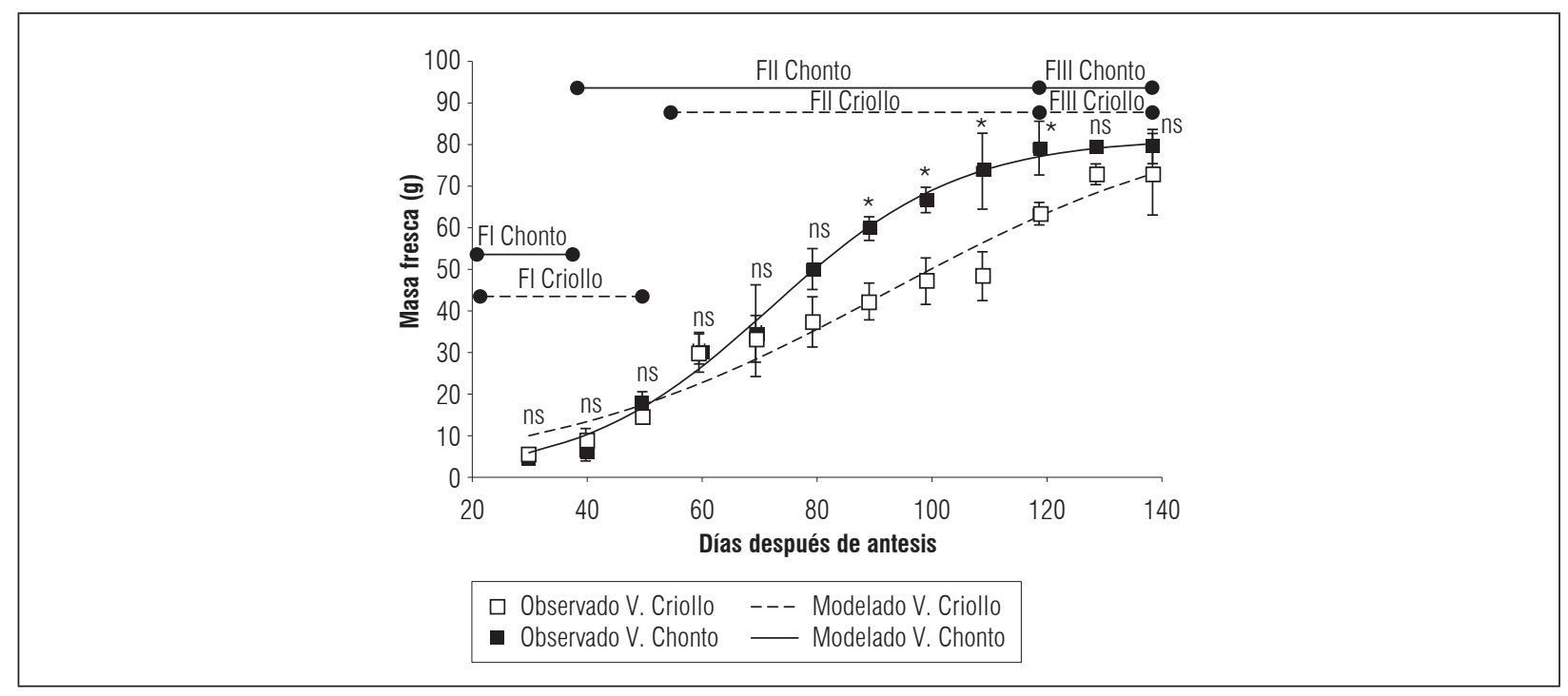

Figura 1. Comportamiento de la masa fresca durante el crecimiento y desarrollo de frutos de lulo variedad Chonto grueso y Criollo en el municipio de Pachavita, Boyacá. *Diferencias significativas al 5\%; ns: no hay diferencias estadísticas. Las barras verticales indican el error estándar $(n=4)$. FI. División celular; F II. Elongación celular; F III. Maduración.

Teniendo en cuenta que los frutos maduros de las dos variedades presentaron un peso menor de $100 \mathrm{~g}$, se clasificaron como calidad cuarta, según las normas clombianas del Icontec (2002). Valores que coinciden con las investigaciones sobre distintos materiales de de lulo (Arizala et al., 2011; González et al., 2014; Jurado et al., 2013), quienes reportan un peso promedio de fruto de entre 80 y $139 \mathrm{~g}$, sin embargo, difiere con Ardila et al. (2015), quienes reportan frutos de mayor tamaño, afirmando que la producción de frutos pequeños, son consecuencia de un mayor número de frutos/planta, que es lo que ocurre normalmente en la zona.
De otra parte, se encontraron diferencias significativas al 5\% entre los 90 y 110 dda debido a que la variedad Chonto superó a la Criollo en los valores de acumulación de masa fresca, sugiriendo un mayor crecimiento en los frutos de la variedad Chonto lo cual puede estar relacionado con el poder vertedero y la acumulación de agua, teniendo en cuenta que la mayoría de la materia seca que se acumula en los frutos, proviene de los fotoasimilados producidos en las hojas, acorde con la relación fuente/vertedero; por tanto, los frutos representan vertederos de alto poder (Casierra-Posada et al., 2007). Se ha sugerido que en tomate (Solanum lycopersicon Mill., cv. Zuisyu), el 
ácido abscísico estimularía la descarga de asimilados en el fruto, lo que promueve la actividad vertedero en los primeros días de su crecimiento (Kojima, 2005), lo cual podría explicar el incremento en la masa fresca de los frutos de lulo en las FI y FII. De igual manera, las auxinas y giberelinas estarían implicadas en el crecimiento activo del fruto, ya que están relacionadas positivamente con el tamaño (Chitarra y Chitarra, 2005).

\section{Firmeza del fruto}

La firmeza se ajustó a un modelo polinómico de orden 4 para ambas variedades (Tab. 1), donde la variedad Chonto grueso mostró su máximo valor de $82,67 \pm 8,09 \mathrm{~N}$ a los $100 \mathrm{dda}$, momento a partir del cual se redujo la firmeza hasta alcanzar un valor en el momento de la cosecha (110 dda) de $58 \pm 0,12 \mathrm{~N}$ y a partir de ese momento disminuyo hasta $0,3 \pm 0,028$ $\mathrm{N}$. Por su parte, la variedad Criollo alcanzó su máximo valor a los 90 dda de 75,93 $\pm 7,42 \mathrm{~N}$, llegando a la cosecha con valor de $61,83 \pm 0,13 \mathrm{~N}$, y a partir de los 110 dda se redujo hasta llegar a un valor de $0,17 \pm 0,03$ $\mathrm{N}$ (Fig. 2), lo que implica que estas variedades tienen una corta vida poscosecha, y por tanto dificultad en la comercialización. Este comportamiento es típico en los frutos, con tendencia a disminuir con el almacenamiento (García et al., 2012) y coincide con los trabajos realizados por Muñoz (2010) que reporta valores cercanos a $61 \mathrm{~N}$ en diferentes cultivares, el estado de madurez 5.

Se ha reportado que la pérdida de firmeza y ablandamiento de frutos es debida a los procesos de degradación de almidón y solubilización de sustancias pécticas, siendo la poligalacturonasa una de las enzimas implicadas en el ablandamiento (Torres et al., 2012), esta enzima cataliza la ruptura hidrolítica de los enlaces de galacturónidos en la matriz de pectina (Villavicencio et al., 2004). Además, la hidrólisis de las paredes celulares en frutos dirigida por enzimas, es el punto de partida para la maduración de frutos climatéricos (Casierra-Posada et al., 2004). Al respecto, Escribano (2003) reporta que la pérdida de firmeza debida al incremento en la solubilidad de la pectina no se debe únicamente a la acción de enzimas, pues se sabe que dicha degradación también es el resultado de los cambios en la concentración de ácidos orgánicos, pérdida de agua del fruto o concentración en el pH de las paredes celulares.

\section{Sólidos solubles totales}

El comportamiento de los SST durante el crecimiento de la variedad Chonto grueso y Criollo se ajustó a un modelo polinómico de orden 3 (Tab. 1). A los $70 \mathrm{dda}$, la variedad criollo inicio con 4,05 $\pm 0,2^{\circ}$ Brix y la variedad chonto con $4,3 \pm 0,2^{\circ}$ Brix, mostrando

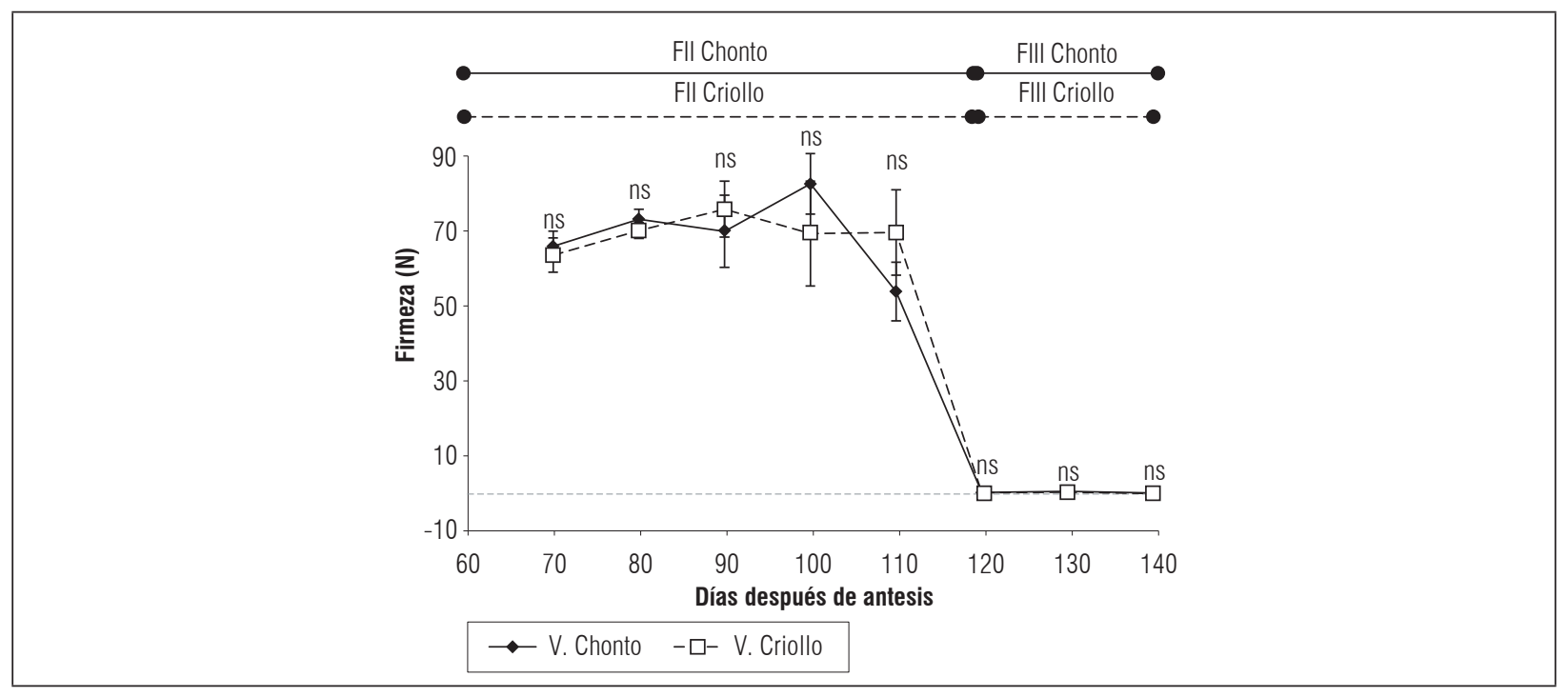

Figura 2. Comportamiento de la firmeza (N) durante el crecimiento y desarrollo de frutos de lulo variedad Chonto grueso y Criollo en el municipio de Pachavita, Boyacá. *Diferencias significativas al 5\%; ns: no hay diferencias estadísticas. Las barras verticales indican el error estándar $(n=4)$. FI. División celular; F II. Elongación celular; F III. Maduración. 


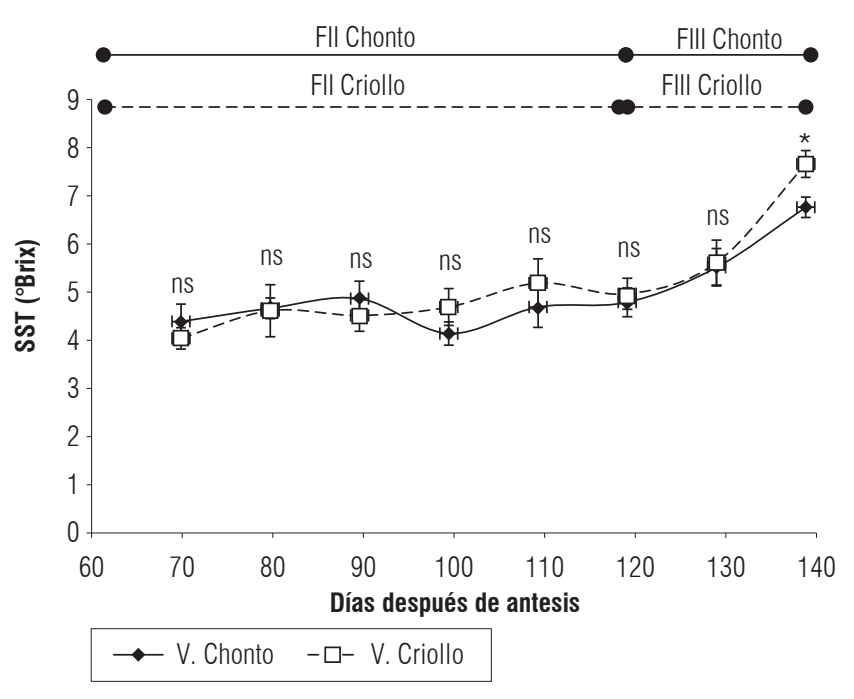

Figura 3. Comportamiento de los sólidos solubles totales (SST) durante el crecimiento y desarrollo de frutos de lulo variedad Chonto grueso y Criollo en el municipio de Pachavita, Boyacá. * Diferencias significativas al $5 \%$; ns: no hay diferencias estadísticas. Las barras verticales indican el error estándar $(n=4)$. FI. División celular; F II. Elongación celular; $\mathrm{F}$ III. Maduración.

un incremento en forma lenta hasta los 120 dda en las dos variedades, hasta el inicio de la FIII, en donde alcanzaron $4,5 \pm 0,2$ y $5,1 \pm 0,2^{\circ}$ Brix respectivamente, a partir de este momento, los valores aumentaron hasta la cosecha con $6,7 \pm 0,2^{\circ} \mathrm{Brix}$ para la variedad Chonto grueso, y $7,6 \pm 0,28{ }^{\circ} \mathrm{Brix}$ para la variedad Criollo, se registraron diferencias estadísticas al $5 \%$, indicando mayor contenido de ${ }^{\circ}$ Brix en la variedad Criollo (Fig. 3).

En frutas tropicales se reporta que los SST aumentan en proporción directa con el proceso de maduración (Almeida et al., 2011). Comportamiento similar, se presentó con las dos variedades, la variedad Criollo, a los 140 dda obtuvo 7,6 $\pm 0,28^{\circ}$ Brix y la variedad Chonto 6,7 $\pm 0,27^{\circ}$ Brix. Múltiples estudios han evaluado la dinámica de los SST en frutos. Reina et al. (1998) reportaron valores de $7,6^{\circ} \mathrm{Brix}$ en frutos de lulo. Muñoz (2010) evaluó 42 materiales de lulo provenientes de distintas localidades, y encontró que los SST variaron entre 5,6 a 12,9. Jurado et al. (2013) evaluaron lulos injertados de la var. quitoense y septentrionale en patrones de otras solanáceas encontrando que los SST tienen un valor promedio de $6,7^{\circ} \mathrm{Brix}$.

Casierra-Posada et al. (2004) y Fischer et al. (2012b) afirman que la cantidad de azúcares en el fruto puede depender de la especie, de la tasa asimilatoria de las hojas, de la cantidad de hojas/fruto, del clima durante el crecimiento del fruto y de la madurez, lo que en parte explicaría el incremento en los SST en etapas finales de los frutos. Lo cual es confirmado por Kays (2004) y Menéndez et al. (2006) al afirmar que el incremento de la concentración en SST de los frutos cuando están madurando, se debe posiblemente a la translocación de la sacarosa desde las hojas, por hidrólisis de almidón y polisacáridos en las paredes celulares produciendo azúcares solubles que, según Gómez et al. (2002) y Fisk et al. (2006) aumentan durante la maduración de los frutos climatéricos como resultado de la actividad enzimática, como por ejemplo la sacarosa fosfato sintasa (SPS).

\section{Acidez total titulable}

La ATT se ajustó a un modelo polinómico de orden 5 para las dos variedades (Tab. 1), a los 70 dda, la variedad criollo presentó un valor de $1,04 \pm 0,15 \%$ y la variedad Chonto $0,4 \pm 0,05 \%$, a partir de este momento, se presentó un comportamiento ascendente, para las dos variedades, hasta los 120 dda (FIII), alcanzando los mayores valores $(3,61 \pm 0,19 \%$, para la variedad Chonto grueso, y $3,84 \pm 0,16 \%$ para la variedad Crio1lo), a partir de ese momento se presenta un descenso y estabilización en los valores hasta el momento de la cosecha (Fig. 4). En ninguno de los puntos de muestreo se presentaron diferencias estadísticas. 


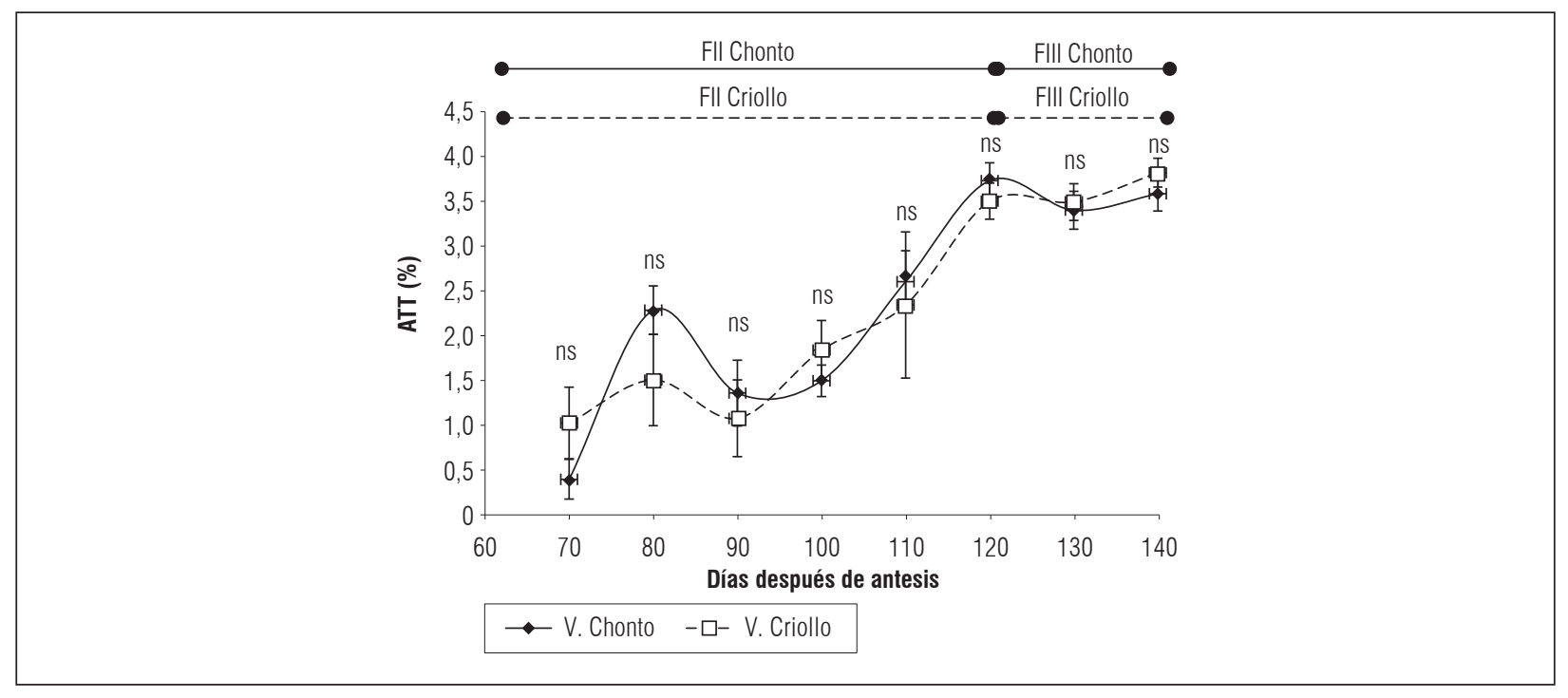

Figura 4. Comportamiento de la acidez total titulable durante el crecimiento y desarrollo de frutos de lulo variedad Chonto grueso y Criollo en el municipio de Pachavita, Boyacá. *Diferencias significativas al 5\%; ns: no hay diferencias estadísticas, de acuerdo con el ANOVA. Las barras verticales indican el error estándar $(n=4)$. Fl. División celular; F II. Elongación celular; F III. Maduración.

Resultados similares han sido reportados por Ochoa (2013) en la var. septentrionale y Mejía et al. (2012) en lulo de Castilla, quienes reportan un incremento de la ATT en los primeros estados de desarrollo. Arizala et al. (2011) reportan un valor de acidez de frutos de $3,2 \%$ para Solanum hirtum, $3 \%$ para S. marginatum y $3,2 \%$ para $S$. quitoense Lam.

Pinzón (2000) reporta que el ácido cítrico es el más abundante en frutos de lulo, con concentraciones alrededor de $13 \%$ en jugo de frutos con $75 \%$ de maduración. Además, en el mismo grado de maduración, los ácidos cítrico, málico y oxálico alcanzan su mayor concentración, para después descender. Según González et al. (2014), la reducción en acidez puede ser consecuencia de la degradación de los ácidos orgánicos en los procesos de respiración, mecanismo vital que ocurre durante la etapa de maduración del fruto, esto explicaría el incremento y reducción, en el porcentaje de acidez para las dos variedades evaluadas conforme se dio el proceso de maduración.

De acuerdo con Kays (2004), la ATT disminuye a medida que los frutos aumentan su nivel de madurez. Esto debido a la actividad de las deshidrogenasas y a que los ácidos orgánicos son utilizados como sustratos respiratorios para la síntesis de nuevos componentes durante la maduración. Al respecto, Chitarra y Chitarra (2005) argumentan que en algunos casos hay un aumento de la acidez durante la maduración, lo que puede reforzar el hecho que en frutos de lulo no se haya presentado una disminución de los valores de acidez, sino por el contrario, un aumento marcado hasta los $120 \mathrm{dda}$. Esto puede estar relacionado con la intensidad respiratoria de los frutos evaluados, que al reducir progresivamente desde los $30 \mathrm{dda}$, pudo haber utilizado únicamente los ácidos orgánicos como sustratos en las primeras etapas de desarrollo (Fig. 5); de igual manera, este incremento en los ácidos orgánicos durante la maduración, podrían dar explicación sobre la dinámica de la ATT para las dos variedades. De otra parte, Yahia et al. (2001) sugieren que la baja actividad enzimática del ácido ascórbicooxidasa en los frutos, permite que las concentraciones de vitamina $C$ sean mayores, ya que esta es la responsable de la degradación oxidativa del ácido ascórbico en tejidos vegetales durante el proceso de maduración (Matarazzo et al., 2013), lo que explica lo encontrado en esta investigación.

\section{Intensidad respiratoria}

El comportamiento de la IR durante el crecimiento y desarrollo de frutos de lulo variedad Chonto grueso y Criollo, se ajustó a un modelo polinómico de orden 4 (Tab. 1) para las dos variedades, sin ser significativas. Sin embargo, se observó una alta tasa respiratoria a los 30 dda de $268,1 \pm 62 \mathrm{mg} \mathrm{CO}_{2} \mathrm{~kg}^{-1} \mathrm{~h}^{-1}$ para la variedad Chonto grueso, y de 215,29 $\pm 81,67 \mathrm{mg} \mathrm{CO}_{2} \mathrm{~kg}^{-1}$ 


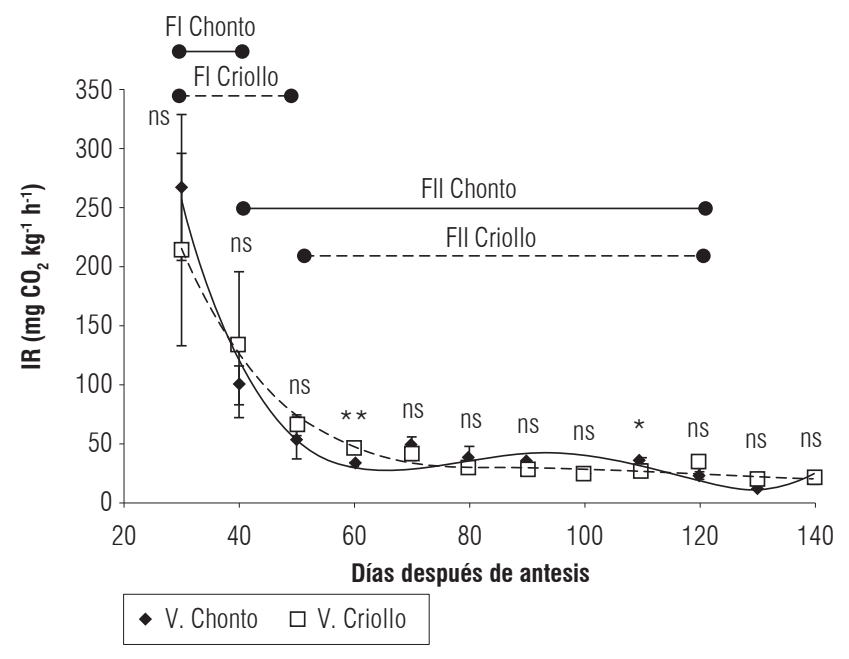

Figura 5. Comportamiento de la tasa respiratoria durante el crecimiento y desarrollo de frutos de lulo variedad Chonto grueso y Criollo en el municipio de Pachavita, Boyacá. *Diferencias significativas al 5\%; ns: no hay diferencias estadísticas. Las barras verticales indican el error estándar $(n=4)$. F I. División celular; F II. Elongación celular; F III. Maduración.

$\mathrm{h}^{-1}$ para la variedad Criollo (Fig. 5), lo cual se presenta en frutos jóvenes por su activo crecimiento en fases tempranas (Balaguera-López y Herrera, 2012). Desde este momento, se presentó una reducción rápida, llegando a $32,69 \pm 8,01 \mathrm{mg} \mathrm{CO}_{2} \mathrm{~kg}^{-1} \mathrm{~h}^{-1}$ para la variedad Chonto grueso, y de 46,21 $\pm 81,12 \mathrm{mg} \mathrm{CO}_{2} \mathrm{~kg}^{-1} \mathrm{~h}^{-1}$ para la variedad Criollo, lo cual es común en la segunda fase de crecimiento de los frutos (Wachoawicz y Carvalho, 2002) hasta los 60 dda y, a partir de entonces presentó disminución. La variedad Chonto grueso tuvo un comportamiento con picos de incrementos a los 70, 110 y $140 \mathrm{dda}$, mientras que la variedad Criollo incrementó a los 120 dda y 140 dda. A los 110 dda, se presentaron diferencias significativas al $5 \%$, en este punto, el aumento en la respiración en la variedad Chonto grueso sugiere el inicio del climaterio; de igual manera, a los 120 dda se mostró un leve incremento en la respiración en la variedad Criollo. La TR al momento de la cosecha fue de 20,53 $\pm 2,7 \mathrm{mg} \mathrm{CO} \mathrm{kg}^{-1}$ $\mathrm{h}^{-1}$ para la variedad Chonto grueso, y de 20,15 $\pm 4,16$ mg CO $\mathrm{kg}^{-1} \mathrm{~h}^{-1}$ para 'Criollo'. Forero et al. (2014) obtuvieron valores de intensidad respiratoria en frutos de lulo que oscilaron entre 5 y $40 \mathrm{mg}$ de $\mathrm{CO}_{2} \mathrm{~kg}^{-1} \mathrm{~h}^{-1}$. Ochoa (2013) reportó un comportamiento similar en la var. septentrionale, con incremento a los $180 \mathrm{dda}$, indicando el inicio del climaterio.

Teniendo en cuenta que los azúcares y los ácidos orgánicos son compuestos importantes en el metabolismo y que son la base para la calidad organoléptica en frutos, y que además, son los principales sustratos respiratorios (Kays, 2004), se explicaría la dinámica existente entre la TR de los frutos de las variedades evaluadas, las cuales mostraron altos valores al inicio del estudio, en tanto, los SST y ATT presentaron bajos valores inicialmente, pues posiblemente fueron utilizados como sustratos para sintetizar nuevas sustancias; y un subsiguiente aumento de los mismos mientras se dio la reducción de la TR. Con ello, queda confirmado que en la respiración, los compuestos altamente energéticos (ATP) y reductores $\left(\mathrm{NADH}_{2}\right)$ son formados a partir de la oxidación de carbohidratos, para ser utilizados en la síntesis de nuevas sustancias (Galho et al., 2007) durante el desarrollo del fruto.

\section{CONCLUSIONES}

Se determinaron tres fases fenológicas en las dos variedades: una fase de división celular con duración de 40 días para la variedad Chonto grueso, y 50 para la variedad Criollo, donde se evidenció un crecimiento lento; una fase de elongación celular, hasta los 110 dda para las dos variedades, con aumento exponencial en la masa fresca y cambios notorios en la dinámica de las propiedades químicas; y una fase de maduración, hasta los 140 dda para las dos variedades.

La masa fresca del fruto de lulo alcanzó su máximo valor entre los 110 y 120 dda, y la firmeza, comenzó 
un fuerte descenso a partir de los $110 \mathrm{dda}$, lo que implica que estas variables fueron afectadas por el grado de madurez. Por el contrario, los SST aumentaron, mientras que la acidez disminuyó levemente.

\section{REFERENCIAS BIBLIOGRÁFICAS}

Agronet. 2015. Ministerio de Agricultura y Desarrollo Rural de Colombia, Análisis-Estadísticas, lulo. En: http:// www.agronet.gov.co; consulta: junio de 2016.

Almeida, A., J. Reis, D. Santos, T. Veira y M. Oliveira. 2011. Estudio de la conservación de la papaya (Carica papaya L.) asociado a la aplicación de películas comestibles. Rev. Venez. Cienc. Tecnol. Aliment. 2(1), 49-60.

Andrade D., D., M. Córdoba, H. Criollo y T. Lagos Burbano. 2013. Evaluación de medios de cultivo para propagación in vitro de semillas y explantes de especies silvestres de Solanum. Acta Agron. 62 (1), 27-36.

Andrade, M., C. Moreno, A. Concellón y B. Chicaiza. 2013. Efecto hormético de la radiación UV-C sobre el desarrollo de Rhizopus y Phytophthora en naranjilla (Solanum quitoense Lam). Rev. Iber. Tecnología Postcosecha 14(1), 64-70.

AOAC. 1995. Official methods of analysis of AOAC International. Association of Official Analytical Chemists. Arlington, VA.

Ardila, G.H., G. Fischer y J.C. García. 2015. La poda de tallos y racimos florales afecta la producción de frutos de lulo (Solanum quitoense var. septentrionale). Rev. Colomb. Cienc. Hortic. 9(1), 24-37. Doi: 10.17584/ rcch.2015v9i1.3743

Ardila, G.H., G. Fischer y H.E. Balaguera-López, 2011. Caracterización del crecimiento del fruto y producción de tres híbridos de tomate (Solanum lycopersicum L.) en tiempo fisiológico bajo invernadero. Rev. Colomb. Cienc. Hortic. 5(1), 44-56. Doi: 10.17584/ rcch.2011v5i1.1252

Arias, F. y S. Rendón. 2014. Inteligencia de mercados para la cadena del lulo (Solanum quitoense). J. Agric. Anim. Sci. 3(2), 38-47.

Arizala, M., A. Monsalvo, C. Betancourth, C. Salazar y T. Lagos. 2011. Evaluación de solanaceas silvestres como patrones de lulo (Solanum quitoense Lam) y su reacción a Fusarium sp. Rev. Cienc. Agríc. 28(1), 147-160.

Avanza, M., S. Bramardi y M. Mazza. 2008. Statistical models to describe the fruit growth pattern in sweet orange Valencia late. Span. J. Agric. 6(4), 577-585. Doi: 10.5424/sjar/2008064-352

Balaguera-López, H.E. y A. Herrera, 2012. Estudio de algunos cambios bioquímicos durante el crecimiento y hasta la cosecha del fruto de champa (Campomanesia lineatifolia R. \& P. Familia Myrtaceae). Rev. Bras. Frutic. 34(2), 460-468. Doi: 10.1590/S0100-29452012000200019
Barceló, C., G. Rodrigo, G. Sabater y R. Sánchez. 2001. Fisiología vegetal. Ed. Pirámide, Madrid.

Cañizares, A., D. Laverde y R. Puesme. 2003. Crecimiento y desarrollo del fruto de guayaba (Psidium guajava L.) en Santa Bárbara, Estado de Monagas, Venezuela. Rev. Cient. UDO Agríc. 3(1), 34-38.

Casierra-Posada, F., E. García y P. Lüdders. 2004. Determinación del punto óptimo de cosecha en el lulo (Solanum quitoense Lam. var. quitoense y septentrionale). Agron. Colomb. 22(1), 32-39.

Casierra-Posada, F., M. Cardozo y J. Cárdenas. 2007. Análisis del crecimiento en frutos de tomate (Lycopersicon esculentum Mill.) cultivados bajo invernadero. Agron. Colomb. 25(2), 299-305.

Chitarra, M. y A. Chitarra. 2005. Pós-colheita de frutos e hortaliças: fisiologia e manuseio. 2 ed. ESAL-FAEPE, Lavras, Brazil.

Coombe, B. 1976. The development of fleshy fruits. Annu. Rev. Plant Physiol. 27, 207-228. Doi: 10.1146/annurev. pp.27.060176.001231

Escribano, M.I. 2003. Maduración y post-recolección de frutos y hortalizas. Consejo Superior de Investigaciones Científicas, Madrid.

Fischer, G., F. Ramírez y P.J. Almanza-Merchán. 2012a. Inducción floral, floración y desarrollo del fruto. pp. 120140. En: Fischer, G. (ed.). Manual para el cultivo de frutales en el trópico. Produmedios, Bogotá.

Fischer, G., P.J. Almanza-Merchán y F. Ramírez. 2012 b. Source-sink relationships in fruit species. A review. Rev. Colomb. Cienc. Hortíc. 6(2), 238-253. Doi: 10.17584/rcch.2012v6i2.1980

Fisk, C.L., M.R. McDaniel, B.C. Strick y Y. Zhao. 2006. Physicochemical, sensory, and nutritive qualities of hardy kiwifruit (Actinidia arguta) 'Ananasnaya' as affected by harvest maturity and storage. J. Food Sci. 71(3), 204-210. Doi: 10.1111/j.1365-2621.2006.tb15642.x

Forero, N., S. Gutiérrez, R. Sandoval, J.Camacho y M. Meneses. 2014. Evaluación poscosecha de las características del lulo (Solanum quitoense Lam.) cubierto con hoja de plátano. Temas Agrar. (1), 73-85.

Gallo, P. 1996. Manual de fisiología, patología postcosecha y control de calidad de frutas y hortalizas. SENA, Regional Quindío, Armenia, Colombia.

Galho, A., N. Lopes, M. Bacarin y M. Lima. 2007. Composição química e respiração de crescimento em frutos de Psidium Cattleyanum sabine durante o ciclo de desenvolvimento. Rev. Bras. Frutic. 29(1), 61-66. Doi: 10.1590/S0100-29452007000100014.

García, J.C., H.E. Balaguera-López y A.O. Herrera. 2012. Conservación del fruto de banano bocadillo (Mussa a a Simmonds) con la aplicación de permanganato de potasio $\left(\mathrm{KMnO}_{4}\right)$. Rev. Colomb. Cienc. Hortic. 6(2), 161-171. Doi: 10.17584/rcch.2012v6i2.1974 
Gómez, M., F. Lajolo y B. Cordenunsi. 2002. Evolution of soluble sugars during ripening of papaya fruit and its relation to sweet taste. J. Food Sci. 67(1), 442-447 Doi: 10.1111/j.1365-2621.2002.tb11426.x

González, D., L. Ordóñez, P. Vanegas y D. Vásquez. 2014. Cambios en las propiedades fisicoquímicas de frutos de lulo (Solanum quitoense Lam.) cosechados en tres grados de madurez. Acta Agron. 63(1), 1-9. Doi: 10.15446/acag.v63n1.31717

Grange, R. 1996. Crecimiento del fruto. pp. 449-462. En: Azcón- Bieto, J. y M. Talón (eds.). Fisiología y bioquímica vegetal. Interamericana McGraw-Hill, Madrid.

Icontec. 2002. NTC 5093. Frutas frescas. Lulo de Castilla. Especificaciones. Instituto Colombiano de Normas Técnicas y Certificación, Bogotá.

Jurado, J., L. Pérez, T. Lagos y C. Benavides. 2013. Comportamiento agronómico de injertos de lulo de Castilla (Solanum quitoense Lam.) en patrones de Solanum spp. Rev. Ciencias Agríc. 30(1), 54-64.

Kays, S. 2004. Postharvest biology. Exon Press. Athens, GA.

Kojima, K. 2005. Phytohormones in shoots and fruits of tomato; Apoplast solution and seedless fruit. JARO 39(2), 77-81. Doi: 10.6090/jarq.39.77

Marcelis, L. y E. Heuvelink. 2007. Concepts of modeling carbon allocation among plant organs. Functional-structural plant modelling in crop production. Springer, The Netherlands.

Matarazzo, P.H., D.L. De Siqueira, L.C. Salomao, D.F. Da Silva y P.R. Cecon. 2013. Desenvolvimento dos frutos de lulo (Solanum quitoense Lam.) em ViçosaMG. Rev. Bras. Frutic. 35(1), 131-142. Doi: 10.1590/ S0100-29452013000100016

Mazorra, M., A. Quintana, D. Miranda, G. Fischer y M. Chaparro. 2006. Aspectos anatómicos de la formación y crecimiento del fruto de uchuva Physalis peruviana (Solanaceae). Acta Biol. Colomb. 11(1), 69-81.

Mejía, C., D. Gaviria, A. Duque, L. Rengifo, E. Aguilar y A. Alegrías. 2012. Physicochemical characterization of the lulo (Solanum quitoense Lam.) castilla variety in six ripening stages. Vitae 19(2), 157-135.

Menéndez, O., S. Lozano, M. Arenas, K. Bermúdez, A. Martínez y A. Jiménez. 2006. Cambios en la actividad de $\alpha$-amilasa, pectinmetilesterasa y poligalacturonasa durante la maduración del maracuyá amarillo (Passiflora edulis var. Flavicarpa Degener). Interciencia 31(10), 728-733.

Morales, J., F. López, J. Pérez, G. Ríos, D. Echeverry y M. Murillo. 2002. Evaluación agronómica del cultivo del lulo (Solanum quitoense Lam.) en la región central cafetera de Colombia. pp. 319-325. En: Memorias IV Seminario Nacional de Frutales de Clima Frío Moderado. Corpoica, Universidad Pontificia Bolivariana, Medellín, Colombia.

Múnera, G. 2002. Nemátodos asociados con el cultivo del lulo. pp. 135-141. En: Giraldo, M.J. y J.P. Higuera (eds.).
Memorias IV Seminario Nacional de Frutales de Clima Frío Moderado. Corpoica, Universidad Pontificia Bolivariana, Medellín, Colombia.

Muñoz, L. 2010. Evaluación agronómica de materiales de lulo Solanumsp, frutal de alto potencial para zonas tropicales. Tesis de maestría. Universidad Nacional de Colombia, Palmira, Colombia.

Ochoa, L. 2013. Estudio del crecimiento y desarrollo del fruto de lulo (Solanum quitoense Lam.) bajo condiciones agroecológicas del municipio de San Antonio del Tequendama (Cundinamarca). Trabajo de grado. Facultad de Ciencias Agropecuarias, Universidad Pedagógica y Tecnológica de Colombia, Tunja, Colombia.

Opara, L. 2000. Fruit growth measurement and analysis. Hort. Rev. 24, 373-431.

Parra, C. y J. Hernández. 2005. Fisiología postcosecha de frutas. Departamento de Ingeniería Civil y Agrícola, Universidad Nacional de Colombia, Bogotá.

Pinzón, M. 2000. Propiedades físicas de cosecha y poscosecha de frutos de Lulo "La Selva". Memorias del Ber Seminario de Frutales de Clima Frío Moderado. Centro de Desarrollo Tecnológico de Frutales, Manizales, Colombia.

Ramírez, J., I. Aristizábal y J. Restrepo. 2013. Conservación de la mora de castilla mediante la aplicación de un recubrimiento comestible de gel de mucílago de penca de sábila. Vitae 20(3), 172-183.

Reina, C., C. Araujo e I. Manrique. 1998. Manejo poscosecha y evaluación de la calidad del Lulo (Solanum quitoense sp.) que se comercializa en la ciudad de Neiva. Facultad de Ingeniería, Universidad Surcolombiana, Neiva, Colombia.

Salisbury, F. y C. Ross. 1994. Fisiología vegetal. Editorial Iberoamericana, México.

Torres, T., S. Beserra, B. Cordenunsi, F.Gozzo, E. Pilau, F. Lajolo y J. Oliveira. 2012. Proteomic analisys ob banana fruit reveals proteins that are differentially accumulated during ripening. Postharvest Biol. Technol. 70, 5150. Doi: 10.1016/j.postharvbio.2012.04.005

Torres, R., E. Montes, O. Pérez y R. Andrade. 2013. Relación del color y del estado de madurez con las propiedades fisicoquímicas de frutas tropicales. Inf. Tecnol. 24(3), 51-56. Doi: 10.4067/S0718-07642013000300007

Villavicencio, L., S. Blankenship y G. Yencho. 2004. Skin adhesion in sweet potato and its lack of relationship to polygalacturonase and pectinmethylesterase during storage. Postharvest Biol. Technol. 583, 113-119.

Wachowitcz, C.M. y R.I.N. Carvalho (eds.). 2002. Fisiologia vegetal e pós-colheita. Champagnat, Curitiba, Brazil.

Yahia, E.M., M. Contreras-Padilla y G. González-Aguilar. 2001. Ascorbic acid content in relation to ascorbic acid oxidase activity and polyamine content in tomato and bell pepper fruits during development, maturation and senescence. LWT-Food Sci. Techn. 34(7), 452-457. 